University of South Carolina

Scholar Commons

$3-2010$

\title{
Reverberation-Chamber Test Environment for Outdoor Urban Wireless Propagation Studies
}

Helge Fielitz

Kate A. Remley

Christopher L. Holloway

Qian Zhang

Qiong Wu

See next page for additional authors

Follow this and additional works at: https://scholarcommons.sc.edu/elct_facpub

Part of the Digital Communications and Networking Commons, Physics Commons, Signal Processing Commons, and the Systems and Communications Commons

\section{Publication Info}

Postprint version. Published in IEEE Antennas and Wireless Propagation Letters, Volume 9, 2010, pages 52-56.

(c) IEEE Antennas and Wireless Propagation Letters, 2010, IEEE

Fielitz, H., Remley, K., Holloway, C., Zhang, Q., Wu, Q., Matolak, D. (2010). Reverberation-Chamber Test Environment for Outdoor Urban Wireless Propagation Studies. IEEE Antennas and Wireless Propagation Letters, 9, 52-56.

http://dx.doi.org/10.1109/LAWP.2010.2042272 accepted for inclusion in Faculty Publications by an authorized administrator of Scholar Commons. For more information, please contact digres@mailbox.sc.edu. 


\section{Author(s)}

Helge Fielitz, Kate A. Remley, Christopher L. Holloway, Qian Zhang, Qiong Wu, and David W. Matolak 


\title{
Reverberation-Chamber Test Environment for Outdoor Urban Wireless Propagation Studies
}

\author{
Helge Fielitz, Kate A. Remley, Senior Member, IEEE, Christopher L. Holloway, Fellow, IEEE, Qian Zhang, \\ Qiong Wu, and David W. Matolak, Senior Member, IEEE
}

\begin{abstract}
We introduce a test environment to replicate the wellknown "clustering" of reflections in power delay profiles arising from late-time delays and reflections. Urban wireless propagation environments are known to exhibit such clustering. The test setup combines discrete reflections generated by a fading simulator with the continuous distribution of reflections created in a reverberation chamber. We describe measurements made in an urban environment in Denver, CO, that illustrate these multiple distributions of reflections. Our comparison of measurements made in the urban environment to those made in the new test environment shows good agreement.
\end{abstract}

Index Terms-Fading simulator, power delay profile, reverberation chamber, wireless device testing.

\section{INTRODUCTION}

$\mathbf{R}$ ECENT publications have described the use of reverberation chambers for efficient, repeatable, free-field testing of wireless devices [1]-[7], improved channel modeling [8], and simulating specific environments [9]-[11]. By adjusting the quality factor of the reverberation chamber's highly reflective cavity through the addition of RF absorbing material, it is possible to tune the decay time of the chamber to simulate realworld propagation environments. The power delay profile exhibited by reverberation chambers consists of a nearly continuous set of multipath reflections that decay over time (exponentially) within the chamber. For replicating certain environments such as indoor or outdoor-to-indoor radio-wave propagation, the nearly continuous power delay profile created in the reverberation chamber is ideal.

Some propagation environments, however, are better represented by a discrete set of reflections. These environments include both urban and suburban settings, where reflecting structures may be located far from the receiver. In this case, the reflections off a structure or group of nearby structures combine

Manuscript received November 13, 2009; manuscript revised January 05, 2010. Date of publication February 05, 2010; date of current version March 05, 2010. This work was supported in part by the Public Safety Communications Research Lab, Office of Law Enforcement Standards, NIST. Work of the US government not subject to copyright in the United States.

H. Fielitz is with the Technical University of Hamburg-Harburg, Hamburg 21073, Germany.

K. A. Remley and C. L. Holloway are with the Electromagnetics Division, National Institute of Standards and Technology, Boulder, CO 80305 USA (e-mail: remley@boulder.nist.gov).

Q. Zhang, Q. Wu, and D. W. Matolak are with the School of Electrical Engineering and Computer Science, Ohio University, Athens, OH 45701 USA.

Color versions of one or more of the figures in this letter are available online at http://ieeexplore.ieee.org.

Digital Object Identifier 10.1109/LAWP.2010.2042272

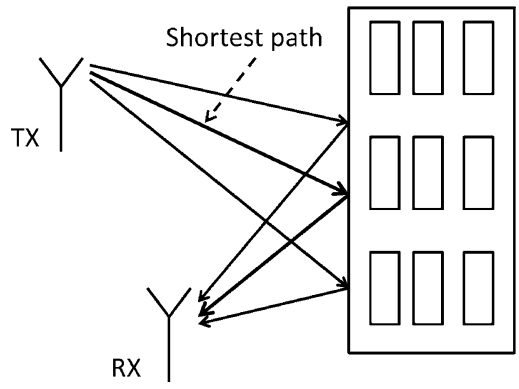

Fig. 1. The origin of the clustered distributions of reflections in an urban environment can be traced to multiple sets of reflections from building structures. Contributions to an individual distribution (cluster) are illustrated here, where the shortest-path reflection will yield the strongest received signal, yet numerous additional reflections will reach the receive antenna as well, all with similar delays.

into essentially one discrete reflection or a collection of discrete reflections. Multiple structures can be represented by multiple discrete reflections. Fading simulators are often used to approximate these discrete-reflection multipath environments. Fading simulators can be used for both conducted testing, where the wireless device is connected through a cable attached to its antenna port, or in free-field testing, where the radiation characteristics including the antenna are measured. For free-field testing, the output of the fading simulator is often fed into an anechoic chamber [12]. The ability of the device's receiver to detect the signal according to performance specifications is then tested.

Unlike the continuously distributed reflections or the discrete reflections described, the power delay profiles from many urban environments exhibit several strong reflections, each of which generates a distribution of nearly continuous reflections [13]. This clustering effect has long been observed in indoor channels as well [14]. This distribution of reflections is created when a signal experiences multiple bounces off a structure, as shown in Fig. 1. This environment is not well represented by either a discrete set of reflections, such as those generated by the fading simulator, or a continuous distribution of reflections, such as those created in a reverberation chamber, but rather combines the two.

We demonstrate a method to replicate such a combined discrete/continuous environment by use of a reverberation chamber and a fading simulator. The fading simulator creates the strongest reflections found in the environment, and the reverberation chamber creates a nearly continuous, exponentially decaying distribution of reflections associated with each strong reflection. 


\section{Power Delay Profile Measurements IN AN URBAN SETTING}

In [15], we described a vector network analyzer (VNA)-based measurement method used to find the power delay profile and RMS delay spread of the outdoor-to-indoor propagation channels associated with various large public structures. In this method, the complex transfer function of the channel is measured as the forward transmission scattering parameter $S_{21}(f)$. The transmitting antenna is connected by a coaxial cable to one port of the VNA, which transmits the test signal. The receiving antenna is connected via a fiber-optic link back to the network analyzer, allowing the two antennas to be separated by long distances (up to $300 \mathrm{~m}$ for the system we used) while maintaining both the magnitude and phase relationships between the transmitted and received signals.

To remove the influence of the optical link and other system effects, the measurement system is calibrated to the short coaxial cables attached to the antenna ports. Using reference measurements made in our laboratory, we de-embed the complex frequency response of the antennas from the measured $S_{21}(f)$ values. We then take the inverse Fourier transform of the measured and corrected transmission parameters to find the power delay profile of the channel. We calculate the RMS delay spread as the square root of the second central moment of the power delay profile as

$$
\tau_{\mathrm{RMS}}=\sqrt{\frac{\int_{0}^{\infty}\left(t-\tau_{0}\right)^{2} P(t) d t}{\int_{0}^{\infty} P(t) d t}} .
$$

By taking several measurements and averaging them, the random component of the measurement uncertainty is reduced. For the measurements reported here, we took 200 repeat measurements. A typical power delay profile is shown in Fig. 2. More detail on the measurement system can be found in [15].

Our test setup was developed as part of a study of the wireless channel encountered in emergency-response scenarios. As a result, the transmitter was located approximately $1.5 \mathrm{~m}$ from the ground in order to simulate an emergency vehicle. While not representative of tower-based cell-phone system installations, the setup is similar to the configuration of many microcell and picocell systems.

In our prior work, we found that most of the outdoor-to-indoor propagation environments exhibited power delay profiles having a nearly continuous, exponentially decaying distribution of reflection, as shown in the example of Fig. 2. However, for non-line-of-sight conditions in the outdoor urban canyon we studied, the power delay profiles typically exhibited the clustering effects described above. To test and verify the performance of wireless devices, it is necessary to replicate this type of power delay profile.

\section{RECREATING THE OUTDOOR URBAN CANYON POWER DELAY PROFILE}

As stated, the reverberation chamber produces a power delay profile that follows an exponentially decaying function. In a reverberation chamber that has been "loaded" with a given amount of RF absorber, the value of the exponent at each paddle position will depend on the relative strength of the various multipath

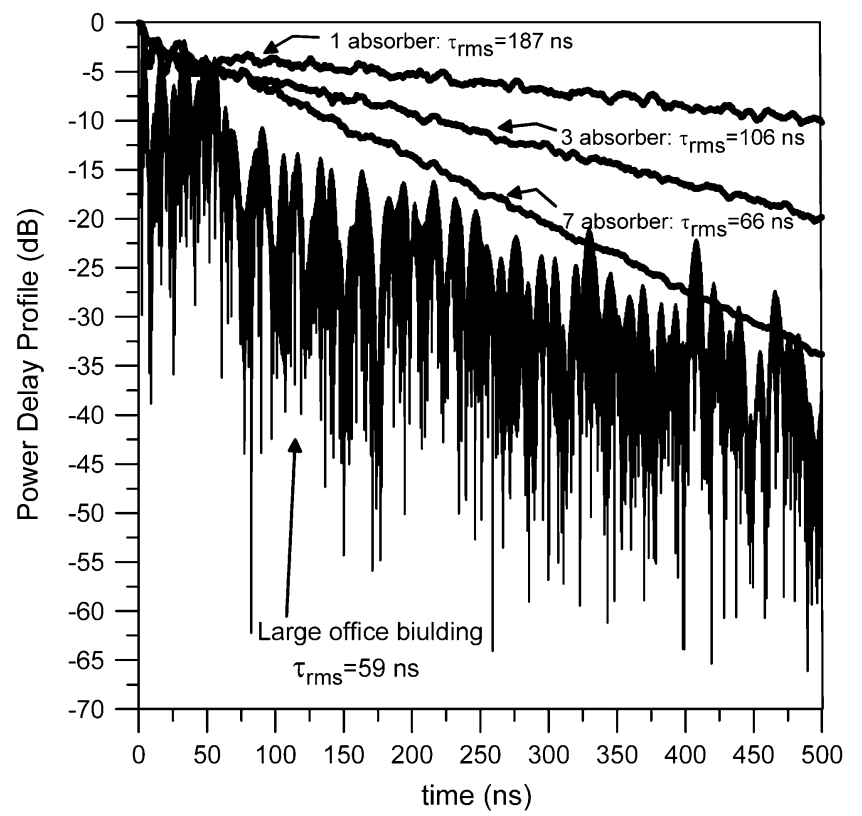

Fig. 2. Power delay profile from NIST measurements of the outdoor-to-indoor propagation channel in a 60-story office building. The lower curve is the instantaneous (thus, jagged) power delay profile measured in the building, and the three upper curves are power delay profiles generated by averaging 100 loaded reverberation chamber measurements [11].

components. We can replicate specific power delay profiles [7], [11] by adding certain amounts of absorber and averaging over many different paddle positions. Inserting a wireless device into an appropriately loaded chamber offers a way of verifying wireless device performance in a repeatable lab-based environment, as shown in Fig. 2. If the transmitter's excitation signal is introduced into the chamber, the ability of the wireless device to receive the channel-impaired signal can be tested.

To create a channel response having multiple discrete clustered distributions, such as those measured in Denver, CO, we feed the transmitter's excitation signal into a fading simulator prior to introducing it into the reverberation chamber, as shown in Fig. 3. By adjusting the amplitude and delay between the fading simulator's discrete components, we can replicate the clustered distributions of reflections seen in the outdoor urban environment.

To tune the characteristics of this test environment to match the characteristics measured in the field, we developed a method to observe the channel's power delay profile in real time. In this method, short RF pulses are fed into the fading simulator with the goal of enabling the user to view the impulse response of the system. We needed to set the duration of the pulses to be long enough to minimize measurement artifacts produced by the fading simulator, yet short enough that each desired multipath cluster could be resolved. For the measurements presented here, for example, we used a pulse duration of $50 \mathrm{~ns}$.

The paddles in the reverberation chamber were set to stir continuously to generate a uniformly distributed field over time. A digital real-time oscilloscope recorded the received signal. The oscilloscope was connected to a PC, where data acquisition and processing programs conducted averaging of the data.

To generate short RF pulses, we used the internal wideband amplitude modulation feature of our vector signal generator. We 


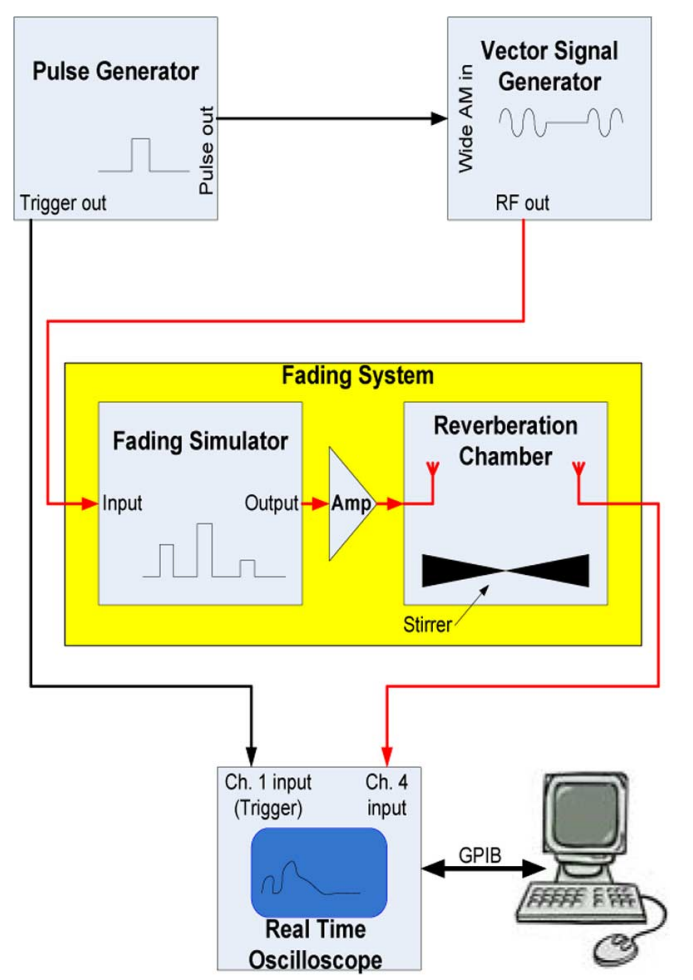

Fig. 3. Test setup for creating the clustered multipath environment in the reverberation chamber. Short pulses are fed into the chamber via the fading simulator. The magnitude and delay of each component from the fading simulator are adjusted until the signal measured at the output of the chamber fits the desired power delay profile.

used a rectangular signal from a pulse generator to vary the modulation index from $0 \%$ to $100 \%$, providing short, "ON-OFF," amplitude-modulated pulses of the carrier signal. A negative bias voltage was fed into the modulator to suppress the output when no signal was present at the modulation input.

The carrier signal produced by the generator is a sinusoidal function, which can be represented as

$$
c(t)=C \sin \left(2 \pi f_{\mathrm{c}} t+\phi_{\mathrm{c}}\right),
$$

where $C$ is the peak amplitude, $f_{\mathrm{c}}$ is the carrier frequency, and $\phi_{\mathrm{c}}$ is the phase of the carrier. The modulation signal, a chain of rectangular pulses, is described as

$$
m(t)=B+A\left\{\begin{array}{cc}
1 & n T \leq t<n T+\Delta t \quad n \in \mathrm{N} \\
0 & \text { otherwise }
\end{array}\right\} .
$$

Here, $\Delta t$ is the duration of one pulse, $B$ is the dc offset of the signal, $T$ is the period, and $A$ is the amplitude of the pulse. The variable $n$ is an index starting with 0 , which describes the position of the pulse in the chain. The output of the AM modulator is the multiplication of the carrier and the modulating signal.

$$
y(t)=(D+m(t)) c(t)
$$

where $D$ is a constant created by the amplitude modulation function. By setting bias $B=-D$ and using (1) and (2), (3) can be simplified to

$$
y(t)=\left\{\begin{array}{cc}
1 & n T \leq t<n T+\Delta t \quad n \in \mathrm{N} \\
0 & \text { otherwise }
\end{array}\right\} A c(t) .
$$

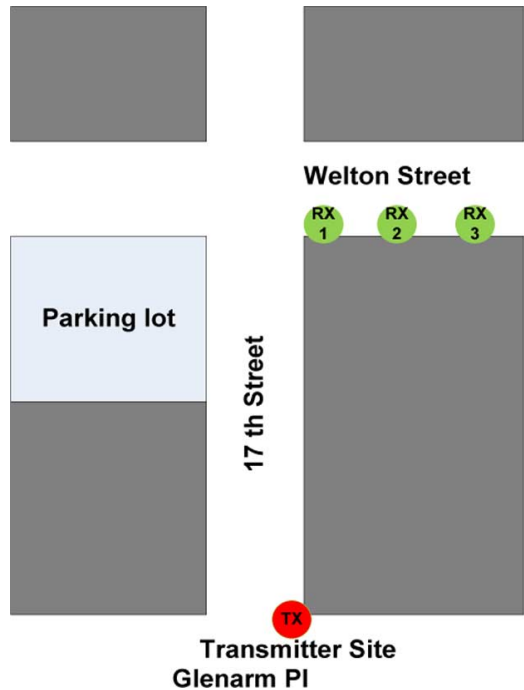

Fig. 4. Diagram of the urban canyon environment where measurements of the clustered power delay profile were collected in a non-line-of-sight condition. The distance from the transmitter to RX1 is approximately $80 \mathrm{~m}$, and the distance between RX1, RX2, and RX3 is $10 \mathrm{~m}$.

In this way, depending on the state of the input pulse, the output can be switched on or off.

We connected an RF amplifier between the fading simulator and the reverberation chamber to boost the signal because the fading simulator we used had a loss of around $25 \mathrm{~dB}$. Driving the fading simulator with a stronger signal at the input was not an option because the instrument would start to clip and add distortion to the signal.

The pulse generator sent a trigger pulse to the oscilloscope to start the data acquisition. At the oscilloscope, a trigger delay was used to compensate for the time taken by the signal to travel through the fading simulator.

\section{MeAsured Results}

Fig. 4 shows an overview of the urban canyon where we measured the power delay profile by use of the method described in Section II. The transmitter and receiver were located in a non-line-of-sight condition. Multistory buildings three or more floors in height were present along both sides of the street except where a flat, single-layer parking lot was located, as indicated in Fig. 4. Most buildings were constructed of glass, steel, and concrete, although a multistory parking structure was located at the upper right corner of Welton and 17th streets.

Fig. 5(a) and (b) show representative measured power delay profiles from our measurements. The solid lines show measurements from downtown Denver, whereas the dashed lines represent measurements made in the combined fading simulator/reverberation chamber test environment. The lower initial values of the received signal, followed by the higher values at later times, indicate that reflections from certain structures may have been stronger than reflections from the structures closest to the receiver.

For the simulated test environment, multiple delayed versions of the pulsed RF signal were generated in the fading simulator 


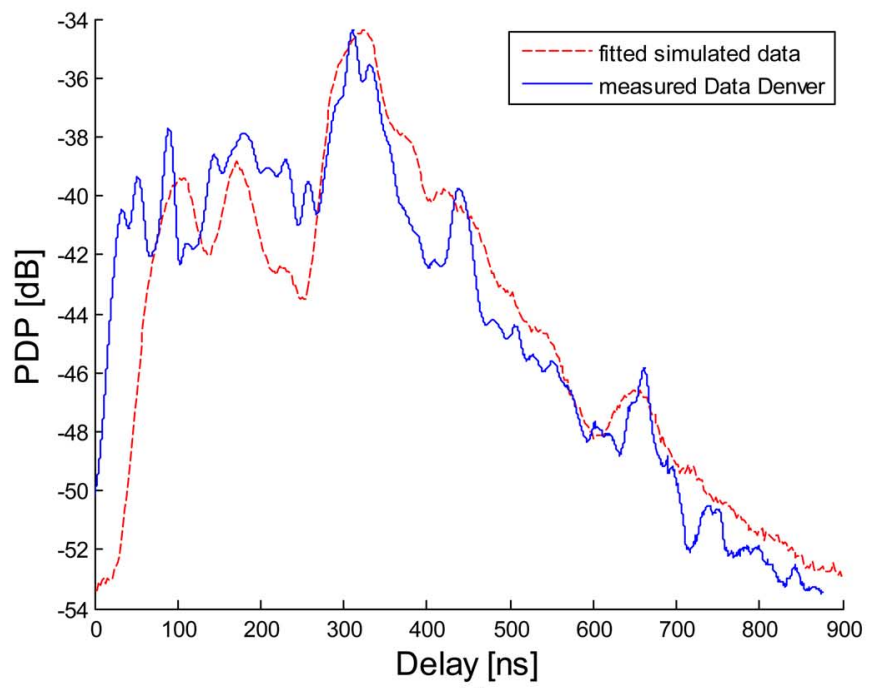

(a)

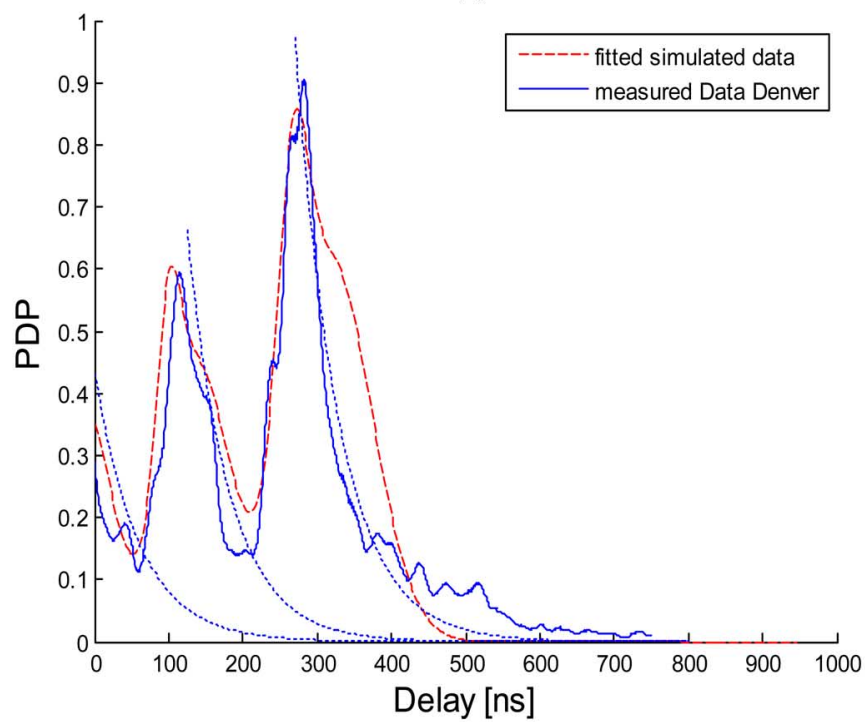

(b)

Fig. 5. Measured power delay profile from the urban environment (solid lines) and from the combined fading simulator/reverberation chamber test environment (dashed lines). (a) TX/RX1 pair. (b) The mean of all non-line-of-sight profiles, RX1 to RX3. In (b), the short dashed lines represent an analytical model (plotted on a linear scale) based on multiple decaying exponential signals.

and fed into the reverberation chamber. The values of the amplitude and delay of each delayed signal were optimized manually to correspond to the measured waveform. In this example, a loading of five RF absorbers produced a RMS delay spread similar to that of the multiple clusters. We determined this value by eye, but a more rigorous technique could easily have been used. Table I shows the delays used in the fading simulator to generate the clustered power delay profile shown in Fig. 5(a).

The short dashed lines in Fig. 5(b) represent exponentially decaying curves based on least-squares fits to analytically model the measured power delay profiles. We model each cluster as

$$
f(\tau)=a \exp (b \tau)
$$

where $\tau$ is the delay. The values of $a$ and $b$ used in our simulations are shown in Table II. The exponential fit supports the use
TABLE I

Values of Delays Used IN THE FAding Simulator to GeNerate the Clustered Power Delay Profile Shown IN Fig. 5(a)

\begin{tabular}{|l|c|}
\hline & delay [ns] \\
\hline Cluster 1 & 100 \\
\hline Cluster 2 & 180 \\
\hline Cluster 3 & 320 \\
\hline Cluster 4 & 420 \\
\hline Cluster 5 & 660 \\
\hline
\end{tabular}

TABLE II

VAlues OF COEFFICIENTS USED IN THE ANALYTIC MODEL OF (5) FOR THE Clustered Power Delay Profile Shown IN Fig. 5(b)

\begin{tabular}{|l|c|c|c|}
\hline & $a$ & $b$ & delay [ns] \\
\hline Cluster 1 & 0.3952 & -0.01685 & 0 \\
\hline Cluster 2 & 6.562 & -0.01774 & 125 \\
\hline Cluster 3 & 129 & -0.01699 & 270 \\
\hline
\end{tabular}

of the reverberation chamber to replicate the individual clusters in the power delay profile.

In Table II, all exponential functions start at time $t=0$, and "delay" describes the time when they are displayed in the plot.

\section{CONCLUSION}

We described a free-field measurement testbed that can be used to test the performance of wireless devices in an outdoor, urban canyon environment that exhibits clustering in the power delay profile. The agreement between field measurements and simulated channels created with the combined fading simulator/ reverberation chamber approach provides a proof of concept for the method described. We anticipate that better agreement could be achieved by use of an optimization algorithm instead of setting the delay taps by inspection. Having recreated the channel corresponding to the field test environment, the user may then insert a wireless device into the reverberation chamber and excite the fading simulator with any desired modulatedsignal waveform for testing receiver performance. We anticipate that this method will be useful for testing and design of wireless devices, including system-level characterization and channel equalizer development and testing.

\section{REFERENCES}

[1] M. Otterskog and K. Madsen, "On creating a nonisotropic propagation environment inside a scattered field chamber," Microw. Opt. Technol. Lett., vol. 43, no. 3, pp. 192-195, Nov. 2004.

[2] P.-S. Kildal and K. Rosengren, "Correlation and capacity of MIMO systems and mutual coupling, radiation efficiency, and diversity gain of their antennas: Simulation and measurement in a reverberation chamber," IEEE Commun. Mag., vol. 42, no. 12, pp. 104-112, Dec. 2004.

[3] C. Orlenius, P.-S. Kildal, and G. Poilasne, "Measurements of total isotropic sensitivity and average fading sensitivity of CDMA phones in reverberation chamber," in Proc. IEEE AP-S Int. Symp., Washington, DC, Jul. 3-8, 2005, vol. 1A, pp. 409-412.

[4] C. L. Holloway, D. A. Hill, J. M. Ladbury, P. F. Wilson, G. Koepke, and J. Coder, "On the use of reverberation chambers to simulate a Rician radio environment for the testing of wireless devices," IEEE Trans. Antennas Propag., vol. 54, no. 11, pp. 3167-3177, Nov. 2006.

[5] C. Orlenius, M. Franzén, P.-S. Kildal, and U. Carlberg, "Investigation of heavily loaded reverberation chamber for testing of wideband wireless units," in Proc. IEEE Antennas Propag. Int. Symp., 2006, pp. 3569-3572. 
[6] O. Delangre, P. De Doncker, M. Lienard, and P. Degauque, "Testing MIMO systems with coupled reverberation chambers: A wideband channel model," in Proc. EuCAP, Nice, France, Nov. 2006, pp. 1-6.

[7] E. Genender, C. L. Holloway, K. A. Remley, J. Ladbury, G. Koepke, and H. Garbe, "Simulating the multipath channel with a reverberation chamber: Application to bit error rate measurements," IEEE Trans. Electromagn. Compat., 2009, submitted for publication.

[8] O. Delangre, P. De Doncker, F. Horlin, M. Lienard, and P. Degauque, "Reverberation chamber environment for testing communication systems: Applications to OFDM and SC-FDE," in Proc. 68th IEEE Veh. Technol. Conf., Sep. 2008, pp. 1-5.

[9] O. Delangre, S. Van Roy, P. De Doncker, M. Lienard, and P. Degauque, "Modeling in-vehicle wideband wireless channels using reverberation chamber theory," in Proc. 66th IEEE Veh. Technol. Conf., 2007, pp. 2149-2153.

[10] J. Cooper, T. Weller, S. DiStasi, and J. Frolik, "An electronically reconfigurable reverberation chamber for the emulation of severe multipath channels," in Proc. WAMICON, Apr. 2009, pp. 1-4.

[11] E. Genender, C. L. Holloway, K. A. Remley, J. Ladbury, G. Koepke, and H. Garbe, "Using reverberation chamber to simulate the power delay profile of a wireless environment," in Proc. EMC Eur., Hamburg, Germany, Sep. 2008, pp. 1-6.
[12] N. Serafimov, P. S. Kildal, and T. Bolin, "Comparison between radiation efficiencies of phone antennas and radiated power of mobile phones measured in anechoic chambers and reverberation chamber," in Proc. IEEE Antennas Propag. Int. Symp., 2002, pp. 478-481.

[13] A. F. Molisch, H. Asplund, R. Heddergott, M. Steinbauer, and T. Zwick, "The COST259 directional channel model-Part I: Overview and methodology," IEEE Trans. Wireless Commun., vol. 5, no. 12, pp. 3421-3433, Dec. 2006.

[14] A. A. M. Saleh and R. A. Valenzuela, "A statistical model for indoor multipath propagation," IEEE J. Sel. Areas Commun., vol. SAC-5, no. 2, pp. $128-137$, Feb. 1987.

[15] K. A. Remley, G. Koepke, C. L. Holloway, C. Grosvenor, D. Camell, J. Ladbury, R. T. Johnk, and W. F. Young, "Radio wave propagation into large building structures; Part 2, Characterization of multipath," IEEE Trans. Antennas Propag., 2009, accepted for publication. 